\title{
Comparison of outcomes: scleral buckling and pars plana vitrectomy versus vitrectomy alone for primary repair of rhegmatogenous retinal detachment
}

This article was published in the following Dove Press journal:

Clinical Ophthalmology

20 December 2016

Number of times this article has been viewed

Luke B Lindsell

Robert A Sisk

Daniel M Miller

Robert E Foster

Michael R Petersen

Christopher D Riemann

Robert K Hutchins

Cincinnati Eye Institute, Department of Ophthalmology, University of

Cincinnati, Cincinnati, OH, USA

Correspondence: Luke B Lindsell

Cincinnati Eye Institute, 1945 CEI Drive,

Cincinnati, OH 45242, USA

$\mathrm{Tel}+\mathrm{I} 5 \mathrm{I} 39845 \mid 33$

Email luke.lindsell@gmail.com
Objective: To assess the combination of scleral buckling (SB) and pars plana vitrectomy (PPV) versus PPV alone in the primary repair of rhegmatogenous retinal detachments (RRDs).

Methods: The current study was a retrospective, comparative, interventional, consecutive case series of 179 eyes of 174 patients who underwent primary RRD repair by five surgeons between January 1, 2008 and December 31, 2010, utilizing SB with PPV or PPV. Univariate and multivariate analyses were used to compare the efficacy of the two surgical strategies and assess for risk factors of proliferative vitreoretinopathy (PVR).

Results: Single surgery anatomic success (SSAS) was similar $(P=0.76)$ between the PPV group (112 of 132 eyes, 85\%) and SB with PPV group (39 of 47 eyes, 83\%). Final anatomic success was $100 \%$ in each group. There was no difference in rates of PVR formation (PPV $16 \%$ vs SB with PPV $19 \%, P=0.70)$. Final logarithm of the minimum angle of resolution acuity was 0.33 (20/43) in the PPV group and $0.37(20 / 47)$ in the SB with PPV group $(P=0.62)$. Postoperative anterior chamber fibrin was highly correlated with PVR formation (PVR 13\% vs no PVR 0.7\%, $P=0.003$; odds ratio $=68.37, P=0.007)$. Separate analysis of medium- to high-complexity cases showed similar SSAS (PPV $86 \%$ vs SB with PPV 83\%, $P=0.45$ ).

Conclusion: SB with PPV versus PPV alone were similarly efficacious for repair of primary RRDs of varying complexity. SSAS rates, PVR incidence, and final visual acuities were not significantly different.

Keywords: scleral buckling, vitrectomy, rhegmatogenous retinal detachment, outcomes, proliferative vitreoretinopathy, comparison

\section{Introduction}

Scleral buckling $(\mathrm{SB})$ is frequently used at the time of primary repair of rhegmatogenous retinal detachment (RRD) by pars plana vitrectomy (PPV) for medium- to highcomplexity RRDs. However, whether the addition of SB to PPV alone increases single surgery anatomic success (SSAS) rate or affects proliferative vitreoretinopathy (PVR) incidence has been under debate. ${ }^{1-15}$ Unfortunately, no prospective, randomized, multicenter study has directly compared PPV versus SB with PPV. The Scleral Buckling versus Primary Vitrectomy in Rhegmatogenous Retinal Detachment Study (SPR) was a prospective, randomized trial to compare these two techniques but did indirectly assess PPV with the addition of SB. ${ }^{1}$ In the PPV arm of the SPR study, SB was performed at the surgeons' discretion, introducing an inherent bias. The authors concluded that pseudophakic patients tended to have a lower rate of recurrent RRD when SB was placed at the time of PPV. ${ }^{1}$ The European Vitreo-Retinal Society Retinal Detachment Study (EVRS) was a large nonrandomized, multicenter retrospective study 
that also sought to clarify surgical strategy for uncomplicated and complex RRD. ${ }^{2,3}$ A supplemental SB was not found to be helpful for uncomplicated RRDs, and patients even had higher failure rates compared with PPV alone. ${ }^{2,3}$ Complex RRDs, however, fared better with PPV alone. ${ }^{3}$ Two recent papers suggested that SB at the time of PPV may improve SSAS for primary RRD repair, although SB did not improve final reattachment rate., ${ }^{4,5}$

The choice of which patients may benefit from a supplemental SB at the time of PPV for primary RRD repair is generally driven by preoperative exam findings and surgeon preferences. Certain characteristics traditionally associated with higher risk for primary surgical failure have been used to justify placement of SB at the time of PPV, including extensive lattice degeneration, large detachment size, phakic lens status, pre-existing PVR, and inferior breaks. ${ }^{4,5,12,14,16}$ However, other evidence suggests that a supplemental SB offers no additional benefit over PPV alone in these situations. , $3,9,10,15,17$ Given this unresolved controversy in the medical literature, we performed a retrospective case review with secondary case-control series to examine whether utilization of SB at the time of PPV improved the SSAS for medium- to highcomplexity RRDs or helped reduce the incidence of recurrent retinal detachment $(\mathrm{RD})$ when PVR developed.

\section{Methods}

Institutional Review Board (Cincinnati Children's Hospital, Cincinnati, OH, USA) approval was obtained for this retrospective, comparative, chart review. The research was conducted in accordance with the Declaration of Helsinki and the Health Insurance Portability and Accountability Act regulations. The Institutional Review Board did not require written informed consent be obtained from the participants, as this was a retrospective study, and all data was anonymous.

Cases were obtained from the Cincinnati Eye Institute, a large multidisciplinary ophthalmology practice in Cincinnati, $\mathrm{OH}$, predominantly serving patients in Ohio, Indiana, and Kentucky. All retinal detachment repairs performed by five vitreoretinal surgeons between January 1, 2008 and December 31, 2010, were reviewed. We identified patients who were diagnosed with a primary RRD repaired by either PPV or SB with PPV. Inclusionary criteria also consisted of a minimum of 6 months of follow-up and an age $>18$ years. Excluded from this analysis were patients with: prior retinal detachment repair, pneumatic retinopexy, prior vitrectomy, grade D PVR, aphakia, unplanned violation of posterior capsule, lensectomy (including phacoemulsification), or intraocular lens (IOL) removal at the time of vitrectomy, proliferative retinal vascular disease, endophthalmitis, presence of intraocular foreign body, giant retinal tears, precipitating ocular trauma, prior posterior uveitis, other intraocular surgery within 90 days prior, dense vitreous hemorrhage $(2+$ or greater), Fluorouracil-Lovenox infusion, methotrexate infusion, $27 \mathrm{G}$ surgery, endoscopic surgery, incomplete records, and $<6$ months of follow-up.

Between the dates listed above, 768 patients were identified who received retinal detachment repair. However, only 179 consecutive eyes from 174 patients met the inclusion criteria. The primary outcome measure was SSAS of RRD repair. Recorded preoperative data consisted of: presence of PVR and grade, lens status, whether the fellow eye had a retinal tear or detachment, inferior retinal breaks (defined as breaks between 4 and 8 o'clock), six or more total clock hours of lattice degeneration, posterior vitreous detachment, status of macula, myopia over 6 diopters, and extent of the RRD measured in clock hours. Best-corrected visual acuity (BCVA) and intraocular pressure (IOP) were recorded at the following intervals after surgery: 1 day, 1 week, 1 month, 3 months, 6 months, and at most recent follow-up. BCVA was converted to the logarithm of the minimum angle of resolution $(\log M A R)$. Count fingers or hand motion vision test results were recorded as a $\log$ MAR value of +2.0 and +3.0 , respectively. ${ }^{18}$ Analyzed postoperative data consisted of recurrent $\mathrm{RD}$, time to recurrence, presence of PVR and grade, return to surgery within 90 days, number of surgeries needed for successful reattachment, IOP over $25 \mathrm{mmHg}$ in the postoperative period, anterior chamber inflammation $>2+$ (standardization of uveitis nomenclature classification), ${ }^{19}$ anterior chamber fibrin (standardization of uveitis nomenclature classification), choroidal/subretinal hemorrhage, need for narcotics, macular edema (assessed by optical coherence tomography), and whether a cataract developed following surgery. Additional analysis was performed on detachments of medium-high complexity, which we defined as the presence of preoperative PVR (except for grade D), inferior retinal detachments, and retinal detachments in two or more quadrants. ${ }^{3,5,17}$

The five vitreoretinal surgeons involved in this analysis performed standard three-port 20G, 23G, or 25G PPV with assistance from a wide-angle noncontact viewing system $\left(\mathrm{BIOM}^{\circledR} 3\right.$; Oculus, Port St Lucie, FL, USA). The vitrectomy systems used were the Accurus $^{\circledR}$ or Constellation ${ }^{\circledR}$ (Alcon, Ft Worth, TX, USA). Endolaser was used to surround all retinal breaks. All surgeries utilized an intraocular tamponade agent of either air, nonexpansile gas $\left(20 \% \mathrm{SF}_{6}\right.$ or $\left.14 \% \mathrm{C}_{3} \mathrm{~F}_{8}\right)$, or 1000 centistokes silicone oil (SILIKON ${ }^{\circledR}$ 1000, Alcon). Every $20 \mathrm{G}$ sclerotomy was sutured with 7-0 vicryl sutures, whereas $23 \mathrm{G}$ and $25 \mathrm{G}$ sclerotomies were sutured only if they 
leaked at the conclusion of the surgery. The encircling element consisted of an encircling 41 band and 70 Watzke sleeve for those patients also receiving SB at the time of PPV. The $\mathrm{SB}$ was placed either before or after the vitrectomy portion of the surgery depending on the surgeon preference.

Data were collected and compiled in a spreadsheet (Excel ${ }^{\circledR}$ 2011; Microsoft Inc., Redmond, WA, USA). Statistical analysis was performed with SPSS version 21.0 (IBM Corp., New York, NY, USA). Chi-square, Fisher's exact test, and independent samples $t$-test were used as appropriate with a $P$-value $<0.05$ considered statistically significant. Multivariate logistic regression was used to control for multiple independent variables potentially affecting the outcome between PPV and SB with PPV. The odds ratio (OR) and probability were calculated from the multivariate analyses and were considered significant if the $P$-value was $<0.05$.

\section{Results}

\section{Demographic and preoperative findings, surgical instrumentation}

This analysis included 179 eyes of 174 patients. One hundred thirty-two eyes received PPV alone (PPV group) and 47 eyes underwent SB with PPV (SB with PPV group) for primary repair of RRD. Demographic and preoperative data are described in Table 1. Regarding the entire cohort, patients in the SB with PPV group more frequently had preoperative PVR (36\% vs $10 \%, P \leq 0.01)$, inferior breaks ( $49 \%$ vs $14 \%$, $P \leq 0.01)$, lattice encompassing $>6$ clock hours of retina ( $21 \%$ vs $5.3 \%, P \leq 0.01$ ), a detached macula ( $76 \%$ vs $55 \%$,
$P=0.01)$, and larger detachments (6.4 clock hours vs 4.5 clock hours, $P<0.01)$. Table 2 summarizes the instrumentation and tamponade agent used.

\section{Anatomical success, postoperative findings and complications - all data}

SSAS rates were similar between PPV and SB with PPV ( $85 \%$ vs $83 \%, P=0.76$ ). Rates of recurrent detachments did not differ between the two groups ( $16 \%$ vs $17 \%, P=0.96$; $\mathrm{OR}=0.65, P=0.54)$. Final anatomical success was $100 \%$ in both groups at the last follow-up visit. PVR was the cause of recurrent retinal detachment in 17 of the 22 cases $(77 \%)$ in the PPV group and six of the eight cases $(75 \%)$ in the SB with PPV group, respectively $(P=0.659)$. The remainder were secondary to either unrecognized or new retinal breaks.

Postoperative findings and complications are listed in Table 3. Univariate and multivariate analysis, respectively, and associated statistical significance values given for each variable are described. There were no significant differences in postoperative findings save except for macular edema, which had a greater frequency in the SB with PPV group ( $28 \%$ vs $11 \%, P=0.005 ; \mathrm{OR}=3.39, P=0.01)$.

\section{Lens status, inferior retinal breaks, preoperative PVR, and SSAS}

Phakic patients were more likely to receive SB with PPV compared with pseudophakic patients $(P=0.20)$ but this was not significant. There was no difference between PPV and SB with PPV for SSAS based on lens status. Patients with

Table I Demographic and preoperative exam findings for PPV versus SB with PPV

\begin{tabular}{|c|c|c|c|}
\hline & PPV, $\mathbf{N}=132$ & SB with PPV, N=47 & $P$-value \\
\hline Age (years), mean & 61 & 58 & 0.055 \\
\hline Sex & & & 0.68 \\
\hline Men, n (\%) & $90(70)$ & $31(67)$ & \\
\hline Women, n (\%) & $38(30)$ & $15(33)$ & \\
\hline Follow-up (months), mean & 28 & 32 & 0.198 \\
\hline Preoperative PVR, n (\%) & $13(10)$ & $17(36)$ & 0.000033 \\
\hline Lens & & & 0.20 \\
\hline Phakic, n (\%) & $50(38)$ & $27(57)$ & \\
\hline Intraocular lens, $\mathrm{n}(\%)$ & $82(62)$ & $20(43)$ & \\
\hline Retinal break or detachment in fellow eye, $\mathrm{n}(\%)$ & $38(29)$ & $17(36)$ & 0.346 \\
\hline Inferior breaks, n (\%) & $19(14)$ & $23(49)$ & 0.000002 \\
\hline Lattice ( $>6$ clock hours), n (\%) & $7(5.3)$ & $10(21)$ & 0.001 \\
\hline Posterior vitreous detachment, $\mathrm{n}$ (\%) & $127(96)$ & $44(93)$ & 0.434 \\
\hline Macula & & & 0.01 \\
\hline Off, n (\%) & $73(55)$ & $36(76)$ & \\
\hline On, n (\%) & $59(45)$ & II (24) & \\
\hline Myopia ( $>6$ diopters), $\mathrm{n}^{*}$ & 29 & 9 & 0.849 \\
\hline Retinal detachment size (clock hours), mean & 4.5 & 6.4 & 0.000001 \\
\hline
\end{tabular}

Note: *Myopia status unknown in 38 PPV and 16 SB with PPV cases.

Abbreviations: PPV, pars plana vitrectomy; PVR, proliferative vitreoretinopathy; SB, scleral buckling. 
Table 2 Surgical instrumentation and tamponade agent for PPV versus SB with PPV

\begin{tabular}{|c|c|c|c|}
\hline & PPV, $\mathbf{N}=132$ & SB with PPV, N=47 & $P$-value \\
\hline \multicolumn{4}{|l|}{ Vitrectomy gauge } \\
\hline 20G, n (\%) & $48(36)$ & $27(57)$ & 0.012 \\
\hline $23 G, n(\%)$ & $63(48)$ & $20(43)$ & 0.54 \\
\hline $25 G, n(\%)$ & $21(16)$ & 0 & 0.001 \\
\hline \multicolumn{4}{|l|}{ Tamponade } \\
\hline Air, n (\%) & $6(4.5)$ & 0 & 0.3425 \\
\hline $20 \% \mathrm{SF}_{6}, \mathrm{n}(\%)$ & $93(70.5)$ & $24(5 \mathrm{I})$ & 0.016 \\
\hline $14 \% \mathrm{C}_{3} \mathrm{~F}_{8}, \mathrm{n}(\%)$ & $5(4)$ & $12(26)$ & 0.00007 \\
\hline I000 cs silicone oil, n (\%) & $28(2 I)$ & II (23) & 0.75 \\
\hline
\end{tabular}

Abbreviations: PPV, pars plana vitrectomy; SB, scleral buckling.

inferior breaks or preoperative PVR attained similar SSAS in both groups $(P=0.709$ and $P=0.705$, respectively).

\section{Proliferative vitreoretinopathy}

Table 4 summarizes the correlation between PVR and several preoperative and postoperative exam findings. Postoperative PVR was more likely if preoperative PVR was also observed ( $35 \%$ vs $13 \%, P=0.006$ ). PVR was highly associated under both univariate and multivariate analyses with regard to recurrent retinal, macular edema, and anterior chamber fibrin. The proportion of surgical failures due to PVR $(68 \%$ for PPV and 66\% for SB with PPV) was similar in both groups $(P=0.659)$. The frequency of PVR was not related to the size of the detachment or whether PPV or SB with PPV was performed.

\section{Visual acuity and IOP outcomes}

Table 5 summarizes preoperative, postoperative, and final recorded visual acuity and IOP. Presenting visual acuity was $\log$ MAR 1.03 in the PPV group and $\log$ MAR 1.19 in the SB with PPV group, which was not significantly different $(P=0.34)$. The final recorded visual acuity was similar for both the groups (PPV 20/43 vs SB with PPV=20/47, $P=0.618$ ). IOP was similar at the initial visit ( $P P V=14 \mathrm{mmHg}$ vs $\mathrm{SB}$ with $\mathrm{PPV}=14 \mathrm{mmHg}, P=0.614)$ and for the final recorded IOP (15 $\mathrm{mmHg}$ vs $14 \mathrm{mmHg}, P=0.255$ ).

\section{Subgroup analysis of medium- to high- complexity rhegmatogenous retinal detachments}

A subgroup analysis of pre- and postoperative results was performed between PPV versus SB with PPV with less complex cases excluded from the PPV arm (Table 6). There were a total of 59 eyes in the PPV arm of moderate-severe complexity based on the preoperative findings versus 47 eyes in the SB with PPV group. The choice between PPV versus SB with PPV was not statistically significant in the presence of preoperative PVR, inferior breaks, lattice degeneration

Table 3 Analysis of postoperative findings for PPV versus SB with PPV

\begin{tabular}{|c|c|c|c|c|c|c|}
\hline & $\begin{array}{l}\text { PPV, } \\
\mathbf{N}=132\end{array}$ & $\begin{array}{l}\text { SB with PPV, } \\
N=47\end{array}$ & $\begin{array}{l}\text { Univariate, } \\
\text { P-value }\end{array}$ & Odds ratio & $95 \% \mathrm{Cl}$ & $\begin{array}{l}\text { Multivariate, } \\
P \text {-value }\end{array}$ \\
\hline Failed primary surgery, $n$ & 0 & 0 & 1.0 & - & - & - \\
\hline Recurrent retinal detachment, n (\%) & $22(16)$ & $8(17)$ & 0.96 & 0.65 & $0.168-2.53$ & 0.54 \\
\hline \multicolumn{7}{|l|}{ Surgeries to attach } \\
\hline One, n (\%) & $112(85)$ & $39(83)$ & 0.76 & & & \\
\hline Two, n (\%) & II (8) & $8(17)$ & 0.11 & & & \\
\hline Three, n (\%) & $5(4)$ & 0 & 0.328 & & & \\
\hline Four, n (\%) & $4(3)$ & 0 & 0.57 & & & \\
\hline PVR formation, n (\%) & $22(16)$ & $9(19)$ & 0.70 & 1.090 & $0.28-4.25$ & 0.90 \\
\hline Intraocular pressure over 25 mmHg, n (\%) & $53(40)$ & $21(44)$ & 0.59 & 1.098 & $0.54-2.24$ & 0.79 \\
\hline Macular edema, n (\%) & $14(11)$ & $13(28)$ & 0.005 & 3.39 & I.34-8.54 & 0.01 \\
\hline Postoperative cataract, n (\%) & $47(35)$ & $23(49)$ & 0.143 & $\mathrm{I} .75$ & $0.87-3.52$ & 0.12 \\
\hline $\mathrm{A} / \mathrm{C}$ inflammation $>2+, \mathrm{n}(\%)$ & II (8) & $4(8)$ & 1.0 & 1.068 & $0.28-4.11$ & 0.923 \\
\hline A/C fibrin, $n(\%)$ & $4(3)$ & I (2) & 1.0 & 0.644 & $0.05-7.8$ & 0.73 \\
\hline Endophthalmitis & 0 & 0 & 1.0 & - & - & - \\
\hline Choroidal/subretinal hemorrhage & $5(4)$ & $2(4)$ & 1.0 & 1.43 & $0.24-8.54$ & 0.698 \\
\hline
\end{tabular}

Abbreviations: A/C, anterior chamber; $\mathrm{Cl}$, confidence interval; PPV, pars plana vitrectomy; PVR, proliferative vitreoretinopathy; SB, scleral buckling. 
Table 4 Analysis of postoperative PVR observation

\begin{tabular}{|c|c|c|c|c|c|c|}
\hline & $\begin{array}{l}\text { PVR } \\
(n=31)\end{array}$ & $\begin{array}{l}\text { No PVR } \\
(n=\mid 48)\end{array}$ & $\begin{array}{l}\text { Univariate, } \\
P \text {-value }\end{array}$ & Odds ratio & $95 \% \mathrm{Cl}$ & $\begin{array}{l}\text { Multivariate, } \\
\text { P-value }\end{array}$ \\
\hline Age (years), mean & 63 & 59 & 0.071 & 1.03 & $0.97-1.1$ & 0.292 \\
\hline Preoperative PVR, n (\%) & II (35) & $19(13)$ & 0.006 & 2.81 & $0.52-15.2$ & 0.229 \\
\hline RD size (clock hours), mean & 5.16 & 4.99 & 0.713 & 1.02 & $0.76-1.37$ & 0.908 \\
\hline Type of surgery, n (\%) & & & 0.823 & 0.92 & $0.18-4.85$ & 0.923 \\
\hline PPV & $22(17)$ & $110(83)$ & & & & \\
\hline SB with PPV & $9(19)$ & $38(8 I)$ & & & & \\
\hline Recurrent RD, n (\%) & $23(74)$ & $7(4.7)$ & 0.000000 & 72.53 & $17.1-306.7$ & 0.000000 \\
\hline Intraocular pressure over $25 \mathrm{mmHg}, \mathrm{n}$ (\%) & $16(52)$ & $58(39)$ & 0.231 & 0.54 & $0.14-2.18$ & 0.389 \\
\hline Macular edema, n (\%) & $12(39)$ & $15(10)$ & 0.00028 & 4.97 & $1.13-21.89$ & 0.034 \\
\hline Postoperative cataract, n (\%) & II (35) & $59(40)$ & 0.691 & 0.55 & $0.13-2.42$ & 0.431 \\
\hline $\mathrm{A} / \mathrm{C}$ inflammation $>2+, \mathrm{n}(\%)$ & $6(19)$ & $9(6)$ & 0.026 & 1.61 & $0.19-13.38$ & 0.658 \\
\hline $\mathrm{A} / \mathrm{C}$ fibrin, $\mathrm{n}(\%)$ & $4(13)$ & $\mathrm{I}(0.7)$ & 0.003 & 68.37 & $3.21-1,457.6$ & 0.007 \\
\hline Choroidal/subretinal hemorrhage, n (\%) & $2(6.5)$ & $5(3)$ & 0.349 & 0.68 & $0.05-8.96$ & $0.77 \mid$ \\
\hline
\end{tabular}

Abbreviations: A/C, anterior chamber; $\mathrm{Cl}$, confidence interval; PPV, pars plana vitrectomy; PVR, proliferative vitreoretinopathy; RD, retinal detachment; SB, scleral buckling.

spanning $>6$ clock hours, macular detachment status, or for size of the detachment. The lens status was the only statistically significant difference between PPV versus SB with PPV where PPV was performed more frequently when an IOL was present ( $63 \%$ vs $43 \%, P=0.039$ ).

Postoperative exam findings were similar between the two groups (Table 7). SSAS was not statistically different and the rate of recurrent detachments did not differ between the two groups. Final anatomical success was $100 \%$ in both groups. No difference was found between PPV and SB with PPV with regard to PVR formation, elevated IOP, macular edema, postoperative cataract formation, anterior chamber inflammation $>2+$, anterior chamber fibrin, or choroidal/ subretinal hemorrhage.

Preoperative vision was $\log$ MAR 1.44 in the PPV group versus $\log$ MAR 1.19 in the SB with PPV arm $(P=0.22)$. BCVA at the final visit was similar between the groups as well. Preoperative IOP between PPV and SB with PPV was

Table 5 PPV versus SB with PPV - VA and IOP

\begin{tabular}{|c|c|c|c|c|c|c|}
\hline & \multicolumn{3}{|c|}{ VA (logMAR) } & \multicolumn{3}{|c|}{ IOP $(\mathrm{mmHg})$} \\
\hline & PPV & $\begin{array}{l}\text { SB with } \\
\text { PPV }\end{array}$ & $P$-value & PPV & $\begin{array}{l}\text { SB with } \\
\text { PPV }\end{array}$ & $P$-value \\
\hline Preoperative & 1.03 & 1.19 & 0.340 & 14.14 & 13.78 & 0.614 \\
\hline Day I & 2.15 & 2.39 & 0.052 & |7.7| & 23.66 & 0.000 \\
\hline Week I & 1.27 & 1.91 & 0.000 & 16.30 & 17.00 & 0.484 \\
\hline Month I & 0.57 & 0.99 & 0.000 & 19.06 & 15.72 & 0.002 \\
\hline Month 3 & 0.45 & 0.70 & 0.006 & 14.78 & 14.98 & 0.788 \\
\hline Month 6 & 0.39 & 0.55 & 0.082 & 13.99 & 13.70 & 0.658 \\
\hline Final & 0.33 & 0.37 & 0.618 & $|4.5|$ & 13.87 & 0.255 \\
\hline Final (Snellen) & $20 / 43$ & $20 / 47$ & & & & \\
\hline
\end{tabular}

Abbreviations: IOP, intraocular pressure; logMAR, logarithm of the minimum angle of resolution; PPV, pars plana vitrectomy; SB, scleral buckling; VA, visual acuity. comparable (14 mmHg vs $14 \mathrm{mmHg}, P=0.8$ ) as was last measured IOP (15 mmHg vs $14 \mathrm{mmHg}, P=0.34$ ).

\section{Discussion}

The debate concerning if and when to add a scleral buckle during PPV for RRD repair has centered around several preoperative factors, such as the presence of inferior breaks, lens status, extent of the detachment, and presence of PVR - with each of these favoring the addition of an encircling element. ${ }^{4,16}$ Surgeons have several effective options for primary RRD repair, including SB alone, $\mathrm{PPV}$ alone, SB with PPV, and pneumatic retinopexy. The preferred technique for RRD generically and specifically has been extensively discussed and debated in the literature, but the trend has leaned toward PPV alone for repair of primary RRD., ${ }^{9,11,20-23}$

One of the first articles suggesting that vitrectomy alone was sufficient in RRD repair came from Escoffery et al in 1985. ${ }^{20}$ Twenty-nine patients underwent 20-gauge PPV with fluid-air exchange for primary RRD with SSAS of 79\%. Phakic, aphakic, and pseudophakic eyes were included in the study. Subsequent publications have confirmed the excellent visual and anatomic success of PPV without SB for repair of primary RRD in phakic and pseudophakic patients. $.15,17,23-25$ In the past decade, several studies have examined whether the addition of SB during a planned PPV increases the SSAS but to date no prospective study has investigated this topic. The majority of these investigations have shown no significant differences in SSAS ${ }^{22,26,27}$ or for SSAS based on lens status, ${ }^{2,10,22,26}$ while two have shown better SSAS for SB with PPV in phakic patients. ${ }^{4,12}$ Nearly all reports regarding $\mathrm{RRD}$ repair in the literature are retrospective in nature, with the notable exception of the SPR study. However, combined 
Table 6 Subanalysis for matched preoperative exam findings - PPV versus SB with PPV

\begin{tabular}{|c|c|c|c|}
\hline & PPV, $\mathbf{N}=\mathbf{5 8}$ & SB with PPV, $N=47$ & $P$-value \\
\hline Preoperative proliferative vitreoretinopathy, n (\%) & $13(22)$ & $17(36)$ & 0.10 \\
\hline Lens & & & 0.039 \\
\hline Phakic, n (\%) & $22(37)$ & $27(57)$ & \\
\hline Intraocular lens, $\mathrm{n}(\%)$ & $37(63)$ & $20(43)$ & \\
\hline Retinal break or detachment in fellow eye, $n$ (\%) & $14(24)$ & $17(36)$ & 0.162 \\
\hline Inferior breaks, n (\%) & $19(32)$ & $23(49)$ & 0.08 \\
\hline Lattice ( $>6$ clock hours), n (\%) & $7(12)$ & $10(21)$ & 0.18 \\
\hline Posterior vitreous detachment, $\mathrm{n}(\%)$ & $57(97)$ & $44(93)$ & 0.653 \\
\hline Macula & & & 0.87 \\
\hline Off, n (\%) & $46(78)$ & $36(76)$ & \\
\hline On, n (\%) & $13(22)$ & II (24) & \\
\hline Myopia ( $>6$ diopters), $\mathrm{n}^{*}$ & 14 & 9 & 0.797 \\
\hline Retinal detachment size, clock hours, mean & 5.9 & 6.4 & 0.33 \\
\hline
\end{tabular}

Note: *Myopia status unknown in 15 PPV and 16 SB with PPV cases.

Abbreviations: PPV, pars plana vitrectomy; SB, scleral buckling.

SB with PPV was not randomized in the trial, creating a secondary nonrandomized endpoint. ${ }^{1}$ A large number of PPV patients in both the phakic and pseudophakic arms $(50.7 \%$ and $66.7 \%$, respectively) received an encircling element. Interestingly, the redetachment rate for PPV versus SB with PPV was similar for the phakic arm but significantly reduced in the pseudophakic arm. ${ }^{1}$ The argument for a supplemental SB in phakic eyes has often centered on the potential difficulty of complete vitreous base shaving. The risk of iatrogenic lens trauma is high during these maneuvers and a supplemental SB could mitigate this risk and potentially increase the SSAS.,11 However, in our series, lens status did not affect SSAS whether or not SB was employed at the time of PPV for primary RRD repair. A recent meta-analysis also found no significant advantage to a supplemental SB in pseudophakic detachments ${ }^{4}$ and the EVRS reached a similar conclusion that a supplemental SB did not enhance the SSAS in pseudophakic or phakic eyes. ${ }^{2}$

PPV and SB with PPV achieved similar SSAS rates ( $85 \%$ and $83 \%$, respectively) and were consistent with previously reported success rates for these techniques. ${ }^{4,10,12,22,26}$ We did not observe an additive benefit to SB over PPV alone for primary RRD repair, but our surgical groups were not initially well matched. Consequently, we performed a secondary analysis matching preoperative characteristics for medium- to high-complexity RRDs and again found similar SSAS. A recent retrospective paper looked at patients at high risk for PVR (defined as RD in two or more quadrants, retinal tears $>1$ hour, preoperative PVR grade $\mathrm{B}-\mathrm{C}$, or vitreous hemorrhage [obscuring 5 or more clock hours of central or peripheral fundus]) who were repaired by either SB with PPV or PPV. ${ }^{5}$ The treatment arms were small but

Table 7 Subanalysis for matched postoperative findings - PPV versus SB with PPV

\begin{tabular}{|c|c|c|c|c|c|c|}
\hline & $\begin{array}{l}\text { PPV, } \\
n=59\end{array}$ & $\begin{array}{l}\text { SB with PPV, } \\
N=47\end{array}$ & $\begin{array}{l}\text { Univariate, } \\
P \text {-value }\end{array}$ & Odds ratio & $95 \% \mathrm{Cl}$ & $\begin{array}{l}\text { Multivariate, } \\
\text { P-value }\end{array}$ \\
\hline Failed primary surgery, $\mathrm{n}$ & 0 & 0 & 1.0 & - & - & - \\
\hline Recurrent retinal detachment, n (\%) & $8(14)$ & $8(17)$ & 0.65 & 1.364 & $0.40-4.71$ & 0.624 \\
\hline \multicolumn{7}{|l|}{ Surgeries to attach } \\
\hline One, n (\%) & $51(86)$ & $39(83)$ & 0.45 & & & \\
\hline Two, n (\%) & $5(9)$ & $8(17)$ & 0.38 & & & \\
\hline Three, n (\%) & $2(3)$ & 0 & 0.50 & & & \\
\hline Four, n (\%) & I (2) & 0 & I & & & \\
\hline PVR formation, n (\%) & $9(15)$ & $9(19)$ & 0.60 & 1.364 & $0.40-4.71$ & 0.624 \\
\hline Intraocular pressure over 25 mmHg, n (\%) & $27(46)$ & $21(44)$ & 0.91 & 0.793 & $0.34-1.84$ & 0.589 \\
\hline Macular edema, n (\%) & $10(17)$ & $13(28)$ & 0.18 & 1.80 & $0.63-5.17$ & 0.276 \\
\hline Postoperative cataract, n (\%) & $20(34)$ & $23(49)$ & 0.12 & 1.78 & $0.79-3.99$ & 0.165 \\
\hline $\mathrm{A} / \mathrm{C}$ inflammation $>2+, \mathrm{n}(\%)$ & $9(15)$ & $4(8)$ & 0.29 & 0.59 & $0.13-2.32$ & 0.422 \\
\hline A/C fibrin, $n(\%)$ & $3(5)$ & I (2) & 0.43 & 0.432 & $0.03-5.73$ & 0.524 \\
\hline Endophthalmitis, $\mathrm{n}$ & 0 & 0 & 1.0 & - & - & - \\
\hline Choroidal/subretinal hemorrhage, n (\%) & I (2) & $2(4)$ & 0.43 & 2.94 & $0.23-38$ & 0.41 \\
\hline
\end{tabular}

Abbreviations: A/C, anterior chamber; $\mathrm{Cl}$, confidence interval; PPV, pars plana vitrectomy; PVR, proliferative vitreoretinopathy; SB, scleral buckling. 
showed an SSAS for SB with PPV of $75 \%$ versus $48.3 \%$ for PPV with the conclusion that a supplemental SB is important for SSAS in these patients. While our own study excluded patients with extensive vitreous hemorrhage and giant retinal tears, other high-risk preoperative characteristics were similar and the addition of SB did not improve the SSAS in our patients. Similarly, the EVRS also reported on complicated RRDs and found that preoperative PVR cases treated with SB with PPV had higher level 1 failure rates (ie, eyes with detached retina judged to be inoperable by the conclusion of the study compared to PPV alone). ${ }^{3}$ Our study had no level 1 failures in either group.

Postoperative complications were similar between the two groups. Only macular edema was more prevalent in the SB with PPV group (28\% vs $11 \%$ in the PPV group). Two recent meta-analyses looking at PPV versus SB did not show any difference in macular edema between the two repair techniques; ${ }^{4,25}$ however, two other reports looking at PPV versus SB with PPV did show increased rates of cystoid macular edema (CME). ${ }^{10,27}$ It is unclear why the SB with PPV arm had more macular edema in our study, but longer surgical time and increased tissue handling during combination surgery have been proposed as possible factors. ${ }^{27}$ Another possible influence, which was not assessed in this study, is laser exposure, which has been associated with a higher incidence of CME. ${ }^{28}$

The rate of PVR formation for the entire PPV group $(17 \%)$ is consistent with that calculated from a recent metaanalysis by Soni et al and from the SPR study. ${ }^{1,25} \mathrm{We}$ found a PVR rate of $19 \%$ in our SB with PPV group, which was statistically insignificant compared to the PPV arm. PVR accounted for nearly all of the recurrent retinal detachments in our study and a concomitant SB did not add protection from this event. However, based on preoperative characteristics for the overall analysis, there was a selection bias favoring placement of SB in more complex cases. A separate subgroup analysis matching preoperative characteristics in each surgical arm yielded no difference in PVR formation and subsequent recurrent detachment.

Additional multivariate analysis looked at postoperative findings and the relationship to PVR. As expected, the development of PVR led to a 72.53 times greater likelihood of developing a recurrent retinal detachment $(P \leq 0.001)$ and a 4.97 times greater likelihood of developing macular edema $(P=0.034)$. The analysis did reveal an interesting correlation between anterior chamber fibrin and PVR (OR =68.37, $P=0.003$ ), highlighting this as a potential independent marker for patients who are at increased risk for postoperative PVR. Increased preoperative aqueous flare in patients with RRD has been associated with higher risk for postoperative PVR. ${ }^{29}$ Few practices own a flare meter, but anterior chamber fibrin may serve as a useful biomarker in counseling patients regarding prognosis for failure of primary RRD repair.

A limitation of our study is the retrospective nature and selection bias for SB with PPV among our full cohort when patients presented with more complex preoperative factors. However, our secondary analysis resulted in no statistical difference for selecting SB with PPV over PPV based on SSAS rates. The small sample size in the SB with PPV arm led to an unbalanced comparison, although the subgroup analysis negated most of this disparity. The extensive exclusion criteria limit the generalizability of the study's conclusions to all RRD scenarios. However, these were purposefully excluded because the hyaloid can be difficult to separate and advance anteriorly in scenarios of trauma, uveitis, and vasoproliferative diseases. A scleral buckle may support the remaining vitreous when the posterior hyaloid face cannot be adequately removed and may be beneficial at preventing recurrent retinal detachment in such cases.

In summary, we found no statistically significant difference in SSAS or long-term visual acuity outcomes between PPV versus SB with PPV for repair of RRD in pseudophakic or phakic patients. "High risk" characteristics (inferior breaks, significant lattice degeneration, and preoperative PVR) had statistically insignificant effects on SSAS whether PPV or SB with PPV was used for repair. Despite the limitations of our study, the statistically insignificant SSAS between the groups supports that PPV alone is effective in most cases of primary RRD. We found no benefit by the addition of an encircling element on the rate of recurrent RD among those patients who developed PVR but did not redetach. Placement of SB poses additional risks, such as globe perforation, choroidal and subretinal hemorrhage, refractive shift, and diplopia, in addition to the increased operating time for placing the SB. Unless a randomized, prospective, multicenter trial sheds new light on this subject, the consensus of level 2 evidence including this study will likely continue to shift the paradigm of primary RRD repair toward PPV alone. The question of whether SB with PPV is better or worse than PPV for any given patient with RRD remains complex and unanswered.

\section{Acknowledgment}

The authors would like to thank Dr Jim Augsburger for his assistance and advice with the statistical analysis for this project.

\section{Disclosure}

The authors report no conflicts of interest in this work. 


\section{References}

1. Heimann H, Bartz-Schmidt KU, Bornfeld N, Weiss C, Hilgers RD, Foerster MH. Scleral buckling versus primary vitrectomy in rhegmatogenous retinal detachment: a prospective randomized multicenter clinical trial. Ophthalmology. 2007;114(12):2142-2154.

2. Adelman RA, Parnes AJ, Ducournau D; European Vitreo-Retinal Society (EVRS) Retinal Detachment Study Group. Strategy for the management of uncomplicated retinal detachments: the European vitreo-retinal society retinal detachment study report 1. Ophthalmology. 2013;120(9):1804-1808.

3. Adelman RA, Parnes AJ, Sipperley JO, Ducournau D; European VitreoRetinal Society (EVRS) Retinal Detachment Study Group. Strategy for the management of complex retinal detachments: the European vitreo-retinal society retinal detachment study report 2. Ophthalmology. 2013;120(9):1809-1813.

4. Totsuka K, Inui H, Roggia MF, Hirasawa K, Noda Y, Ueta T. Supplemental scleral buckle in vitrectomy for the repair of rhegmatogenous retinal detachment: a systematic review of literature and meta-analysis. Retina. 2015;35(11):2423-2431.

5. Storey P, Alshareef R, Khuthaila M, et al. Wills PVR Study Group. Pars plana vitrectomy and scleral buckle versus pars plana vitrectomy alone for patients with rhegmatogenous retinal detachment at high risk for proliferative vitreoretinopathy. Retina 2014;34(10):1945-1951.

6. Wykoff CC, Scwartz SG, Adelman RA, Brucker AJ, Flynn HW Jr. Primary rhegmatogenous retinal detachment repair: evidence supports an individualised approach. Br J Ophthalmol. 2015;99(11):1451-1453.

7. Schwartz SG, Flynn HW, Mieler WF. Update on retinal detachment surgery. Curr Opin Ophthalmol. 2013;24(3):255-261.

8. Rush RB, Simunovic MP, Sheth S, Kratz A, Hunyor AP. Pars plana vitrectomy versus combined pars plana vitrectomy-scleral buckle for secondary repair of retinal detachment. Ophthalmic Surg Lasers Imaging Retina. 2013;44(4):374-379.

9. Colyer MH, Barazi MK, Von Fricken MA. Retrospective comparison of 25 -gauge transconjunctival sutureless vitrectomy to 20 -gauge vitrectomy for the repair of pseudophakc primary inferior rhegmatogenous retinal detachment. Retina. 2010;30(10):1678-1684.

10. Weichel ED, Martidis A, Fineman MS, et al. Pars plana vitrectomy versus combined pars plana vitrectomy-scleral buckle for primary repair of pseudophakic retinal detachment. Ophthalmology. 2006;113(11): 2033-2040.

11. Chong DY, Fuller DG. The declining use of scleral buckling with vitrectomy for primary retinal detachments. Arch Ophthalmol. 2010; 128(9):1206-1207.

12. Mehta S, Blinder KJ, Shah GK, Grand MG. Pars plana vitrectomy versus combined pars plana vitrectomy and scleral buckle for primary repair of rhegmatogenous retinal detachment. Can J Ophthalmol. 2011; 46(3):237-241.

13. Avery RL, Nasir M, Castellarin A, Couvillion S. Retinal detachment repair: A comparison of pneumatic retinopexy, pars plana vitrectomy and scleral buckling within a group private practice. Poster presented at: American Society of Retinal Specialists 29th Annual Meeting; August 21, 2011; Boston, MA.
14. Schneider EW, Geraets RL, Johnson MW. Pars plana vitrectomy without adjuvant procedures for repair of primary rhegmatogenous retinal detachment. Retina. 2012;32(2):213-219.

15. Kinori M, Moisselev E, Shoshany N, et al. Comparison of pars plana vitrectomy with and without scleral buckle for the repair of primary rhegmatogenous retinal detachment. Am J Ophthalmol. 2011;152(2): 291-297.

16. Alexander P, Ang A, Poulson A, Snead MP. Scleral buckling combined with vitrectomy for the management of rhegmatogenous retinal detachment associated with inferior retinal breaks. Eye. 2008; 22(2):200-203.

17. dell'Omo R, Barca F, Tan HS, Bijl HM, Oberstein SY, Mura M. Pars plana vitrectomy for the repair of primary, inferior rhegmatogenous retinal detachment associated to inferior breaks. A comparison of a 25 -gauge versus a 20-gauge System. Graefes Arch Clin Exp Ophthal. 2013;251:485-490.

18. Holladay JT. Visual acuity measurements. $J$ Cataract Refract Surg. 2004;30(2):287-290

19. Jabs DA, Nussenblatt RB, Rosenbaum JT. Standardization of uveitis nomenclature for reporting clinical data. Results of the first international workshop. Am J Ophthalmol. 2005;140(3):509-516.

20. Escoffery RF, Olk RJ, Grand MG, Boniuk I. Vitrectomy without scleral buckling for primary rhegmatogenous retinal detachment. Am J Ophthalmol. 1985;99(3):275-281.

21. Ho JD, Liou SW, Tsai CY, Tsai RJ, Lin HC. Trends and outcomes of treatment for primary rhegmatogenous retinal detachment: a 9-year nationwide population-based study. Eye. 2009;23(3):669-675.

22. Wong CW, Wong WL, Yeo IY, et al. Trends and factors related to outcomes for primary rhegmatogenous retinal detachment surgery in a large Asian tertiary eye center. Retina. 2014;34(4):684-692.

23. Miller DM, Riemann CD, Foster RE, Petersen MR. Primary repair of retinal detachment with 25-gauge pars plana vitrectomy. Retina. 2008; 28(7):931-936.

24. Campo RV, Sipperley JO, Sneed SR, et al. Pars plana vitrectomy without scleral buckle for pseudophakic retinal detachments. Ophthalmology. 1999;106(9):1811-1815.

25. Soni C, Hainsworth DP, Almony A. Surgical management of rhegmatogenous retinal detachment: a meta-analysis of randomized controlled trials. Ophthalmology. 2013;120(7):1440-1447.

26. Orlin A, Hewing NJ, Nissen M, et al. Pars plana vitrectomy compared with pars plana vitrectomy combined with scleral buckle in the primary management of noncomplex rhegmatogenous retinal detachment. Retina. 2014;34(6):1069-1075.

27. Schaal S, Sherman MP, Barr CC, Kaplan HJ. Primary retinal detachment repair - comparison of 1-year outcomes of four surgical techniques. Retina. 2011;31(8):1500-1504.

28. Nonaka A, Kiryu J, Tsujikawa A, et al. Inflammatory response after scatter laser photocoagulation in non-photocoagulated retina. Invest Ophthalmol Vis Sci. 2002;43(4):1204-1209.

29. Schroder S, Muether P, Caramoy A, et al. Anterior chamber aqueous flare is a strong predictor for proliferative vitreoretinopathy in patients with rhegmatogenous retinal detachment. Retina. 2012;32(1):38-42.
Clinical Ophthalmology

\section{Publish your work in this journal}

Clinical Ophthalmology is an international, peer-reviewed journal covering all subspecialties within ophthalmology. Key topics include: Optometry; Visual science; Pharmacology and drug therapy in eye diseases; Basic Sciences; Primary and Secondary eye care; Patient Safety and Quality of Care Improvements. This journal is indexed on Submit your manuscript here: http://www.dovepress.com/clinical-ophthalmology-journal

\section{Dovepress}

PubMed Central and CAS, and is the official journal of The Society of Clinical Ophthalmology (SCO). The manuscript management system is completely online and includes a very quick and fair peer-review system, which is all easy to use. Visit http://www.dovepress.com/ testimonials.php to read real quotes from published authors. 